\title{
Alter
}

Revue de phénoménologie

21 | 2013

La Vie

\section{Vie et risque}

Le végétal au risque du mouvement

Natalie Depraz

\section{(2) OpenEdition}

Journals

Édition électronique

URL : http://journals.openedition.org/alter/795

DOI : 10.4000/alter.795

ISSN : 2558-7927

Éditeur :

Association ALTER, Archives Husserl (CNRS-UMR 8547)

Édition imprimée

Date de publication : 1 novembre 2013

Pagination : 51-71

ISBN : 978-2-95-223749-9

ISSN : 1249-8947

Référence électronique

Natalie Depraz, «Vie et risque », Alter [En ligne], 21 | 2013, mis en ligne le 01 juin 2019, consulté le 05 juillet 2019. URL : http://journals.openedition.org/alter/795 ; DOI : 10.4000/alter.795 


\section{VIE ET RISQUE LE VÉGÉTAL AU RISQUE DU MOUVEMENT ${ }^{1}$}

Natalie Depraz

Besoin de remplacer le risque de la relation par la sécurité de la possession ${ }^{2}$

Ch. Yannaras

\section{Introduction}

Vivre, c'est prendre des risques. Une telle proposition peut paraître triviale. Pourtant, elle va à l'encontre d'une tendance aujourd'hui dominante à « surprotéger » les êtres vivants contre tout danger éventuel, en cherchant à maîtriser le plus possible ce que l'on peut nommer les aléas anxiogènes de notre « avenir ». Qu'on l'appelle prévention (médicale, routière), prospective (managériale), anticipation (financière), orientation (professionnelle), on cherche à minimiser les risques dus à l'incertitude et à l'indétermination du futur en multipliant les programmes d'information et de formation. Pourtant, jamais ces programmes ne parviendront à éliminer tous les dangers - on pensera à la «tuile » spinoziste, et l'argument peut se retourner rapidement, de façon tout aussi problématique: autant ne rien prévoir, et vivre dans l'instant - on pensera au Jacques le Fataliste de Diderot! Un argument un peu plus subtil met en avant le danger plus important encouru par une personne surprotégée (par exemple survaccinée, surencadrée), dès lors incapable de réagir à un danger imprévu et devenant surdépendante du cadre censé la protéger ! Cette

\footnotetext{
${ }^{1}$ Mes remerciements à l'évaluateur de mon article, qui m'a permis par ses remarques utiles de préciser certains aspects conceptuels et argumentatifs de mon propos.

${ }_{2}^{2}$ Ch. Yannaras, Variations sur le Cantique des Cantiques, Paris, Desclée de Brouwer, 1995, p. 26.
} 
personne surprotégée courra un danger plus grand dans une situation inconnue et dans un environnement hostile, où elle devra prendre une décision seule et sans expérience préalable. Sur le fond de cette entente nietzschéenne lato sensu de la vie comme dynamique d'exposition positive à l'inconnu, je voudrais élaborer ici une articulation conceptuelle entre vie et risque. Le fil conducteur que je mettrai à l'épreuve sera le suivant : être vivant, c'est être ouvert et exposé à l'imprévisible, c'est-à-dire c'est risquer (sa vie) à chaque instant.

Lorsque l'on parle de la vie d'êtres singuliers, on a habituellement en tête les êtres vivants humains ou animaux. Et dès lors, la définition la plus courante de la vie passe par le mouvement, entendu dès Aristote dans ses quatre formes principales ${ }^{3}$, rendu central chez Merleau-Ponty avec la primauté de la motricité, voire, plus largement, avec la notion plus ouverte de mobilitét. Ainsi, le « se mouvoir » est devenu dans la tradition phénoménologique (quoiqu'il convienne d'en faire remonter la détermination à Aristote) le trait caractéristique principal du vivant ${ }^{5}$. À la différence de Husserl, qui fait droit dans ce cadre aux « kinesthèses » en tant que qualités sensorielles en mouvement du sujet corporel, mais met au premier plan la perception en tant qu'activité du sujet conscient ou pré-conscient ${ }^{6}$, Merleau-Ponty déplace l'activité première du sujet du côté de la motricité et définit le vivant comme un "mouvant», c'est-à-dire comme un "être mouvement », attribuant une priorité au mouvement sur la perception. Plus fondamentalement encore, si - et ce sera mon hypothèse de départ la vitalité du vivant tient bien à sa mobilité, c'est-à-dire à son être en

\footnotetext{
${ }^{3}$ Aristote, Physique Tome 1, Livre IV, Paris, Les Belles Lettres, 1983, 192b: «[...] chaque être naturel, en effet, a en soi-même un principe de mouvement et de fixité, les uns quant au lieu, les autres quant à l'accroissement et au décroissement, d'autres quant à l'altération. Au contraire, un lit, un manteau et tout autre objet de ce genre, en tant que chacun a droit à ce nom, c'est-àdire dans la mesure où il est un produit de l'art, ne possèdent aucune tendance naturelle au changement, mais seulement en tant qu'ils ont cet accident d'être en pierre ou en bois ou en quelque mixte, et sous ce rapport; car la nature est un principe et une cause de mouvement et de repos pour la chose en laquelle elle réside immédiatement, par essence et non par accident ». ${ }^{4}$ N. Depraz, "Moving without Moving: A first person experiential phenomenological approach », in Moving Imagination. The motor dimension of imagination in the arts, éd. Helena De Preester, Amsterdam, J. Benjamins Publishing Compagny, 2013, et, à titre de phénoménologie appliquée, N. Depraz, F. Mauriac et L. Zeltner, « Les figures de la mobilité dans le travail avec les adolescents : déplacement, mobilisation, créativité », éd. S. Tordjman, Les équipes mobiles auprès des adolescents en difficulté, Paris, Masson, 2009, p. 51-79.

${ }^{5}$ Voir par exemple M. Sheets-Johnstone, The primacy of movement, Amsterdam/Philadelphia, John Benjamins Publishing Compagny, 1991, et R. Barbaras, Le désir et la distance, Paris, Vrin, 2000.

${ }^{6}$ E. Husserl, Chose et espace. Leçons de 1907, Paris, PUF, 1989, quatrième section, « Le concept phénoménologique de kinesthèse ", §45, sixième section, "La constitution du changement objectif », §82, appendice X au §83.
} 
mouvement, et ce, selon des figures différenciées (déplacement, mobilisation, créativité) que j'ai mentionnées plus haut (note 3), lui interdire ou l'empêcher de se mouvoir en ce sens revient à l'anéantir. Pour accompagner sa vitalité voire la développer, il sera déterminant d'encourager sa dynamique motrice, ou encore, dit autrement, d'épouser ses avancées pulsionnelles (tout à la fois mobilisantes et créatrices).

C'est pourquoi, du sein de ce cadre conceptuel, j'ai trouvé utile d'introduire une nouvelle distinction entre deux modes d'être du vivant: d'une part, le mode du risque, d'autre part, le mode de la sécurité. S'agissant de ces deux modes d'être, disons d'entrée de jeu que l'on n'a pas affaire à des données provenant seulement de la nature, comme des récurrences devenant et devenues habitudes, mais à des activités et à des dispositions émanant d'un sujet existant et doté d'une éthique en tant que personne. Cela a pour conséquence que la distinction, modale, entre risque et sécurité, ne sera pas une alternative stricte pour un sujet confronté à l'action (ce qu'il est intrinsèquement), mais se présentera comme un curseur graduel que celui-ci déplace en fonction de son aptitude à s'éprouver comme vivant, mais aussi de sa représentation interne de ce qu'est la vie en tant qu'épreuve. Certes, il y a en chaque sujet une dose d'ouverture au risque, laquelle signe notre désir d'une vie " augmentée » passant par le goût pour le nouveau et l'inconnu, c'est-à-dire d'une vie qu'on dira vécue avec un maximum d'intensité, ou bien, il y aura un souci de sécurité qui relève de la «conservation» de la vie, de ce que l'on appelle le «maintien » en vie, ou encore de la recherche d'une assise et d'une stabilité source de construction de soi, mais tout l'enjeu d'une trajectoire de vie tiendra dans le travail opéré par le sujet sur cette donne initiale inscrite en chacun et polarisée entre risque et sécurité. En d'autres termes, chacun d'entre nous travaille avec ces potentialités, l'accent étant porté davantage sur l'un ou l'autre, et notre force d'incarnation, notre vitalité spécifique - notre mobilité propre - sera un alliage intéressant et subtil, à chaque fois singulier, de sécurité et de risque, de repos et d'aventure, ou encore, pour le dire autrement, de programmation et de surprise ${ }^{7}$. La question étant : comment ces deux modalités de notre vie de vivant en tant que sujet s'agencent-elles en chacun d'entre nous? Qu'est-ce qui fait que l'un

\footnotetext{
${ }^{7}$ Je renvoie, sans pouvoir/vouloir m'y appesantir ici, au programme de recherche en cours « La surprise au sein de la spontanéité des émotions : un vecteur de cognition élargie », ANR Emphiline EMCO (2012-2015), que je pilote aux Archives-Husserl (ENS/CNRS) et, notamment, à la page ANR sur le site de ce centre de recherche.
} 
accordera une primauté au mode du risque et l'autre au mode de la sécurité ?

Tentons, à titre préliminaire et pour tenter de situer et de légitimer cette distinction modale risque/sécurité qui, il faut le dire, n'a pas de précédent conceptuel direct ${ }^{8}$, sa double "traduction », phénoménologique et pragmatique : 1) sur le terrain de la phénoménologie, je lierai le mode du risque au Leib, et le mode de la sécurité au Körper. Pourquoi ? Le Leib contient en lui-même l'ouverture initiale et irréductible de la vie (Leben) en tant que flux indéterminé, là où le Körper renvoie à la conformation structurelle du corps, à savoir à son aspect clos et déterminé ; un corps caractérisé par son ouverture inclut également de la plasticité, ainsi que certains degrés d'incertitude et d'hésitation, c'est-à-dire de liberté ; une morphologie physique déterminée procure une certaine stabilité et du confort, tout autant qu'une forme d'aisance, mais signifie aussi une tendance à l'objectivation, à savoir une aliénation possible ${ }^{9}$; ) au plan pragmatique cognitif, la distinction se complexifie : l'image du corps renvoie en effet à une directionnalité ouverte qui est propre à la dynamique de l'intentionnalité, tandis que le schéma corporel relève du mode de la familiarité et de $l^{\prime}$ habitus ${ }^{10}$. Cependant, même s'ils sont tous deux habités par un certain jeu de plasticité interne, ces deux aspects pragmatiques du corps restent inscrits sous le signe de la "sécurité ». Ils manifestent des dimensions de l'expérience corporelle qui se trouve structurées par l'horizon du monde proche sédimenté et par la présence des autres familiers. À cet égard, ces structures d'inscription dans le monde quotidien et de relation avec l'altérité proche contribuent à réassurer à chaque moment pour moi la stabilité de mon existence corporelle, et relèvent globalement de mon "savoir-faire ». Au contraire, un corps ouvert au risque inhérent à son agir sera traversé par la possibilité à chaque fois imminente de n'être plus, à savoir ouvert à sa mort prochaine : c'est le mode de la précarité/fragilité, remarqua-

\footnotetext{
${ }^{8}$ Certes, cette distinction n'est pas non plus entièrement nouvelle: elle trouverait des alliés évidents chez des auteurs comme Nietzsche, Bergson voire Goldstein.

${ }^{9}$ Voir à ce propos N. Depraz, "La traduction de Leib, une crux phaenomenologica », in Études phénoménologiques, Bruxelles, Ousia, 1997, no 26, p. 91-109.

10 Voir S. Gallagher, «Body Image and Body Schema : A Conceptual Clarification », in Journal of Mind and Behavior 7, 1986, p. 541-554. Je prends cette distinction comme une distinction opératoire utile, qui possède selon moi sa consistance propre dans la cohérence du travail conceptuel de Gallagher, sans préjuger de sa vérité historique textuelle, chez les inventeurs physiologues de ces notions (Shilder, Head) pas plus que dans sa reprise merleau-pontienne stricte. (À ce propos, Emmanuel de Saint Aubert, Être et chair I, manuscrit présenté dans le cadre de l'Habilitation à diriger des recherches, défendue en mai 2013 à l'Université de Paris IV, Promoteur J.-F. Courtine.)
} 
blement esquissé par Hans Jonas dans The Phenomenon of Life, qui signe alors la vitalité du vivant ${ }^{11}$. Plus précisément encore, alors que le savoir-faire du corps forge à mesure des capacités et sédimente le vécu mémoriel et imageant du sujet, conférant ainsi une assise sécurisante à notre mode d'exister, la mobilité incessante du flux vivant définit un corps tout entier dynamique, sans réserve, allant toujours de l'avant, étoile filante toujours au bord de son évanouissement ${ }^{12}$.

Je voudrais dans cette contribution traiter de la complexité de ces deux modes du vivant (risquélsécurisé) qui, depuis le socle conceptuel de la mobilité alias vitalité, signent la qualité de notre épreuve humaine spécifique en tant que vivant. Pour ce faire, je partirai de la distinction comparée standard entre le vivant animal et le vivant végétal, qui me servira de levier "naturaliste », et j'inquiéterai ce plan naturaliste initial en l'investissant phénoménologiquement par les questions suivantes : est-il si sûr, comme le pense Hans Jonas, que la vie animale relève du seul mode du risque et la vie végétale du mode de la sécurité ? Question sous la question : quid du vivant humain ? Entre-til dans cette bipartition et, si oui, est-ce aux côtés du vivant animal ? En partant d'une analyse de cette distinction jonassienne (résiduellement naturaliste), j'entrerai dans une conception moins distinctive, plus intégrée de la vitalité-mobilité du vivant, ce qui pourrait me conduire à proposer une entente reconfigurée du vivant humain et de sa spécificité irréductible à la distinction modale du risque et de la sécurité d'où je suis partie.

\section{Aventure animale, repos végétal}

Il n'est pas besoin de recourir à de grandes analyses philosophiques ou scientifiques pour affirmer que les bêtes sont des vivants qui bougent et se déplacent, tandis que les plantes se tiennent dans une «station » immobile. L'observation immédiate des uns et des autres ne détrompe pas l'affirmation générale: les uns sont des organismes « mouvants », les autres sont «immobiles ». Si vous mettez des plantes dans votre appartement, vous ne vous attendez pas à ce qu'elles se déplacent toutes seules! Votre fille vous supplie de lui offrir un chien

\footnotetext{
${ }^{11} \mathrm{H}$. Jonas, The Phenomenon of Life, toward a philosophical biology, Chicago, The University of Chicago Press, 1966 (Phoenix Edition 1982), trad. D. Lories, Le phénomène de la vie. Vers une biologie philosophique, Bruxelles, De Boeck Université, 2001, Essai 1, « La vie, la mort et le corps dans la théorie de l'être », p. 19-51.

12 Voir à ce propos, N. Depraz, Lucidité du corps. De l'empirisme transcendantal en phénoménologie, Dordrecht, Kluwer, 2001, sections I et II notamment.
} 
comme cadeau d'anniversaire, et vous marquez un temps d'arrêt, car vous savez pertinemment que c'est vous qui allez devoir le sortir deux fois par jour! Ou bien : vous vous promenez sur un chemin de terre en pleine campagne et vous voyez des éperviers qui planent au loin, un machaon qui volette à quelques mètres de vous, tandis que les coquelicots et les feuilles des merisiers oscillent sous la brise. Bref, très simplement, pour vivre, les animaux ont besoin d'aller et venir, ne serait-ce que pour se nourrir, soit en tuant d'autres animaux, soit en consommant des animaux morts ; en revanche, les végétaux ne cherchent pas leur nourriture, ils attendent qu'elle vienne à eux: la pluie qui les nourrit, la terre d'où elles tirent leur substance.

\section{A. La dualité animal/végétal en jeu dans les multiples classifications naturalistes}

Une telle distinction spontanée entre règnes animal et végétal a été, au-delà de l'observation immédiate, thématisée dès l'Antiquité grecque par les philosophes qui se sont intéressés à la classification de la nature. Tout en distinguant les minéraux, êtres inanimés, et les êtres doués de vie, les animés (zên), ils différencient aussi, plus subtilement, les végétaux (zôn) dotés d'une vie simple, et les animaux (zôon) êtres animés non-végétaux, animal, humain et dieux étant tous trois nommés génériquement des «faunes ». Alors qu'Aristote s'est attaché à décrire et à classifier les différentes espèces animales en genre et en espèces, par exemple dans son Histoire des animaux, c'est Théophraste au III e siècle avant notre ère dans son Historia plantarum, puis Dioscoride au I ${ }^{\mathrm{er}}$ siècle après Jésus-Christ dans son Materia Medica et sa description de plus de 600 végétaux, qui donneront toute leur place à l'étude spécifique des végétaux.

Cette conception distinctive entre animaux et végétaux va de pair avec la vision naturaliste d'un continuum entre minéral, vivant et spirituel, ce qui donne sens par contrecoup à la présence de multiples intermédiaires inclassables : les coraux sont des intermédiaires entre le minéral et le vivant, les éponges participent à la fois du végétal et de l'animal. Au XVIII siècle, Carl von Linné perpétuera une telle vision dans son Système de la nature13, en y alliant perméabilité et

\footnotetext{
${ }^{13}$ C. Linnaeus, Systema Naturae sive regna tria naturae, systematics proposita per classes, ordines, genera $\mathcal{E}$ species, $1^{\text {re }}$ édition, 1735, trad., Système de la nature, en trois règnes de la nature, divisés en classes, ordres, genres et espèces, avec les caractères, les différences, les synonymes et les localisations, (titre de la 10e édition de 1758 : dixième édition (1758), Stockholm, sur le site de l'université de Göttingen.
} 
dualité : il formalise un système « binomial » de nomenclature (genre/ espèce) depuis la construction des deux règnes (regna), et envisage en 1767 un "règne chaotique» (regnum chaoticum) pour classer les « animaux-plantes », également nommés « zoophytes » par Trévanius un siècle plus tard. D'où l'émergence au XIX e siècle d'un troisième règne, mixte, avec Ernst Haeckel (1866) et l'existence d'organismes, tel l'euglène, inclassable comme animal ou comme végétal : ce sont les "protistes », qui correspondent à des organismes microscopiques, inférieurs et unicellulaires. En avançant dans sa recherche, Haeckel différenciera les protistes inférieurs, les bactéries par exemple, et les protistes supérieurs, protozoaires, algues, champignons et moisissures $^{14}$.

Ce qui frappe le philosophe dans ces classifications scientifiques qui s'affinent au fil des siècles et à mesure que la connaissance du vivant gagne en précision, c'est la prudence et le souci de décrire méticuleusement ce qui se présente à l'observation externe puis interne du vivant. On n'est donc pas étonné que le raffinement classificatoire se poursuive au $X X^{\mathrm{e}}$ siècle, soit par des re-nominations ${ }^{15}$, soit par différenciation croissante des règnes, soit encore par inclusion en domaines : en 1956, Copeland revendiquera quatre règnes ${ }^{16}$, en 1969 Whitaker propose à son tour une nomenclature à cinq règnes en mettant en évidence trois niveaux d'organisation cellulaire ${ }^{17}$, jusqu'à ce que, à la fin du $X X$ e siècle, le critère de la classification change radicalement, en faisant droit à la phylogenèse au détriment des critères plus subjectifs/observables, morphologiques, anatomiques, écologiques ou comportementaux. Cela conduit à affiner les distinctions entre les organismes les plus archaïques, et à considérer que les séparations les plus anciennes se jouent entre bactéries, archées et eucaryotes, quitte à faire jouer un critère moléculaire et non plus seulement cytologique qui place certaines bactéries (par exemple les archéobactéries) au même niveau phylogénétique que les eucaryotes.

\footnotetext{
${ }^{14}$ E. Haeckel, Generelle Morphologie der Organismen, Berlin, Reimer, 1866.

${ }^{15}$ Aussi É. Chatton, Titres et Travaux Scientifiques (1906-1937), Sottano (Sète, Italie), 1937, baptiset-il les protistes inférieurs " procaryotes » et les supérieurs " eucaryotes », proposant ainsi une classification du monde du vivant en deux types cellulaires (sans/avec noyau).

${ }^{16}$ H. F. Copeland, dans The Classification of Lower Organisms, Palo Alto, Pacific Books, 1956, distingue 1) les mychota (algues bleu-vert et bactéries), les protoctistes (algues eucaryotes, champignons, moisissures et protozoaires), les plantes (embryophytes et algues vertes) et les animaux (incluant les éponges).

${ }_{17}$ R. H. Whittaker, « New concepts of kingdoms of organisms », in Science, 1969, vol. 163, p. 150160, où apparaissent trois « domaines » (procaryote, eucaryote unicellulaire et eucaryote multicellulaire), distingués selon le mode de nutrition: photosynthétique, par absorption et par ingestion.
} 
C'est ce qui conduit Woese, en 1981, à baptiser ces dernières "Archées », et à créer un nouveau plan d'organisation du monde du vivant basé sur un niveau supérieur au règne: le domaine, tout en admettant que, chez les eucaryotes, les règnes des animaux, des plantes et des champignons peuvent être conservés ${ }^{18}$. Pour conclure ce tour d'horizon des avancées classificatrices naturalistes du vivant, mentionnons la recherche récente de Cavalier-Smith (2004), qui suggère à son tour (comme Chatton en 1937) un système à deux "domaines », procaryotes et eucaryotes, mais distribué cette fois en six règnes: tandis que seules les bactéries appartiennent au premier, protozoaires, chromistes, animaux, plantes et champignons relèvent du second ${ }^{19}$.

On peut tirer trois premières conclusions de ces avancées scientifiques impressionnantes dans la connaissance différenciée du vivant : 1) la complexification des différences au sein des règnes ne modifie pas fondamentalement la distinction entre végétal et animal ; 2) cette dernière n'est cependant pas exclusive : on voit apparaître des vivants qui ne sont ni l'un ni l'autre ${ }^{20}$; 3) le vivant n'est plus appréhendé seulement selon des critères observables ou subjectifs, mais se trouve inscrit dans une dynamique " phylogénétique » qui contribue à circulariser sa compréhension : des vivants unicellulaires (les archéobactéries par exemple), situés au même niveau phylogénétique (moléculaire) que les eucaryotes, quoique morphologiquement (cytologiquement) moins développés, possèdent des propriétés phylogénétiques comparables aux vivants les plus évolués. Comment ces acquis naturalistes peuvent-ils retentir sur la reprise "phénoménologique » du vivant que je vais à présent privilégier depuis la distinction des «modes d'apparaître » du vivant animal et du vivant végétal ? ${ }^{21}$ Les critères de la motricité et de l'immobilité (d'entente

\footnotetext{
${ }^{18}$ C. R. Woese \& G. E. Fox, "Phylogenetic structure of the prokaryotic domain: The primary kingdoms », PNAS, 1977, vol. 74, no 11, p. 5088-5090.

${ }^{19}$ T. Cavalier-Smith "A revised six-kingdom system of life », Evolutionary Biology Programme, Canadian Institute for Advanced Research, Department of Botany, University of British Columbia, Vancouver, EC, Canada, 1998, et T. Cavalier-Smith, " Only six kingdoms of life ", Proceeding Research Society, Londres, 2004, p. 1251-1262.

${ }^{20}$ Voir par exemple J. M. Scamardella, « Not plants or animals: a brief history of the origin of Kingdoms Protozoa, Protista and Protoctista », Interior Microbiology, 1999, Vol. 2 (4), p. 207-216.

${ }^{21}$ À propos de l'articulation entre le point de vue phénoménologique en première personne qui excède l'opposition naïve de l'intérieur et de l'extérieur au profit d'une approche du vécu singulier au monde, ici à la vie, et le point de vue naturaliste qui tient les contraintes de l'objectivité (qu'elles soient d'ailleurs externes, morphologique ou comportementale, ou internes, phylogénétique), cf. par exemple Première, deuxième, troisième personne, Rouen, PUHR, à paraître, éd. N. Depraz, volume issu d'un colloque tenu à l'Université de Rouen au printemps 2009.
} 
merleau-pontienne), eux-mêmes déplacés à partir de la distinction modale risque/sécurité (inspirée lato sensu de Jonas), permettent-ils de poser de nouvelles questions aux classifications naturalistes ? Cellesci ouvrent-elles à leur tour des pistes fécondes à même de mettre à l'épreuve les critères phénoménologiques? Bref, notre méthode, encore un peu abstraite à ce stade, se veut "co-générative », dans le sens d'une stimulation mutuelle entre naturalisme et phénoméno$\operatorname{logie} 22$.

Je repartirai pour commencer de la distinction en termes d'apparaître visuo-moteur entre animal et végétal, à quoi se greffent deux modes de nutrition différents, hétéro- ou auto-trophique, ce dernier correspondant à la production de matière organique par réduction de la matière inorganique, alors que le premier ne peut se développer qu'en prélevant de la matière organique dans le milieu. Selon le premier critère de la motricité liée à l'observation à l'œil nu, on placerait les champignons dans la même catégorie que les végétaux, ce que mentionne lui-même L. Baillaux en situant par exemple chanterelle et plantes du même côté et l'hirondelle de l'autre ${ }^{23}$, alors que le critère de la «trophie » place les champignons et les animaux sur le même plan hétérotrophique.

Mais au-delà de l'ambiguïté "phénoménologique »du champignon, déjà notée d'un point de vue naturaliste, il paraît convergent pour phénoménologues et naturalistes de distinguer pour commencer vivant animal et vivant végétal depuis un critère visuo-moteur,

\footnotetext{
${ }^{22}$ Cette méthode co-générative a été proposée pour la première fois par F. J. Varela dans son article pionnier intitulé « Neurophenomenology: A methodological remedy for the hard problem ", in Journal of Consciousness Studies, 1996, n³, p. 330-49. Voir aussi à ce propos : D. Rudrauf, A. Lutz, D. Cosmelli, J.-P. Lachaux, M. Le Van Quyen, «From autopoiesis to neurophenomenology: Francisco Varela's exploration of the biophysics of being ", in Biological Research, 2003, no 36, p. 21-59. Elle apparaît désormais à ce jour comme la méthode intégrative la plus rigoureuse et la plus féconde dans la recherche d'interfaces à la fois conceptuels et expérientiels entre les sciences (neuro, psycho et bio) et la phénoménologie dans sa teneur philosophique, et interroge de façon extrêmement percutante la circulation entre les méthodes en troisième et en première personnes, ce qui produit des effets remarquablement percutants, en retour, sur le nécessaire déplacement des lignes conservatrices de fracture encore existantes entre l'empirique et le transcendantal, soit entre la naturalité et la philosophie. Cf. à ce propos l'article pionnier de F. J. Varela, "The Naturalization of Phenomenology as the Transcendence of Nature; Searching for generative mutual constraints », in Alter, 1997, no 5, p. 355-381, et « When transcendental genesis encounters the naturalization project ", in Naturalizing Phenomenology (J. Petitot, F. J. Varela, J.M. Roy, B. Pachoud éd.), Standford University Press, 2000, trad. N. Depraz, "Quand la genèse transcendantale rencontre le projet de naturalisation ", in Naturaliser la phénoménologie. Essai sur la phénoménologie contemporaine et les sciences cognitives, dir. J. Petitot, F. J. Varela, B. Pachoud et J.M. Roy, Paris, CNRS éditions, p. 609-635.

${ }^{23}$ L. Baillaux, "Critères généraux de la délimitation du monde végétal et rapports entre structures, physiologie et milieu de vie : mise en évidence d'un réseau de déterminismes ? », Bulletin no 25 de la SFBT (mars 2000), p. 35-44.
} 
lequel s'approfondit dans les critères vécus et internes de l'activité motrice et de la passivité réceptive ${ }^{24}$. La question sera: cette distinction est-elle stable, et dans quelle mesure sera-t-elle éprouvée voire interrogée par la profondeur « existentielle» lato sensu de la distinction modale risque/sécurité ?

\section{B. Le mode d'être de l'animal : de la motricité à la prise de risque,} une reprise phénoménologique différenciée de la naturalité ?

L'étymologie nous fournit une première indication instructive: « animal » vient du latin anima, qui signifie « l'âme » et plus avant la " respiration », c'est-à-dire la pulsion motrice vitale au sens primaire de la poussée de vie de l'être à sa naissance, celle-ci même qui fait aussi spontanément émerger la succion chez le nourrisson. Respirer, c'est faire communiquer de façon pré-consciente, à notre insu, l'intérieur et l'extérieur, ce qui forme la base de la connaissance de soi par l'autre (le monde, les autres) et de l'autre par soi ${ }^{25}$. Dès leur naissance, les animaux bougent leur muscles et leurs organes sensoriels (système cardiaque, saccades oculaires, narines, bouche, gueule), leur visage par des mimiques, mais aussi leurs membres (mains, pieds, pattes), assez vite ils se mettent à attraper, à toucher, à saisir, à goûter; ils rampent, marchent, courent. Tous ces mouvements contribuent tout à la fois à la croissance de leur corps et de leur esprit, étant soit strictement nutritionnels soit plus largement, de façon complémentaire, source de connaissance du monde.

Maints auteurs situés à la croisée de la biologie et de la phénoménologie confirmeront cette définition du vivant animal par le critère de la motricité. Pour n'en mentionner que trois, et non des moindres : von Uexküll définit très tôt le mode d'être des animaux comme étant structuré de façon éminente par leur relation mobile à l'environnement. Il forge à cet égard le concept de Umwelt, terme selon lequel chaque espèce vivante a son univers propre, selon une double dynamique de donation de sens par le sujet et d'imposition de contraintes à ce dernier. Renvoyant dos à dos l'introspection, qui ne décrit que les qualités corporelles sensori-motrices vécues, et le comportement, qui ne prend en compte que la conduite observable

\footnotetext{
${ }^{24} \mathrm{Cf}$. à ce propos von Uexküll, Streifzüge durch die Umwelten von Tieren und Menschen - Bedeutungslehre, Hamburg, Rowohlt Verlag, 1956, trad. Mondes animaux et monde humain, Paris, Denoël, Médiations, 1984.

25 À propos de ce sens initial de l'animalité, voir Alter no 3, «L'animal », Dijon, Alter, 1995.
} 
des animaux dans le monde, leurs vécus relevant d'une inaccessible «boîte noire », Uexküll opte pour une voie descriptive médiane, celle du mode d'être de l'animal. À l'exemple de la description de la vie de la tique, petite araignée parasitaire qui ne réagit qu'à trois stimulants (a. olfactif, l'odeur des glandes sudatives des mammifères, b. tactile, le contact avec la peau de ces derniers, c. gustatif, leur sang), l'auteur nous propose une entente interactive du vivant et de son environnement, cette dynamique tout entière formant le «monde » de ce vivant. À cet égard, l'image de $l^{\prime}$ «arc intentionnel» suggère une compréhension relationnelle du vivant animal, caractérisé par une dynamique sensori-motrice circulaire et récursive du corps animal et de son monde environnant. Contrairement à l'attitude atomiste, qui voit les sensations comme des impressions locales qui affectent le corps de l'animal de l'extérieur et s'impriment ponctuellement en elles, Uexküll développe une vision « holiste » de l'organisme animal relié au monde vivant selon une dynamique inter-active.

Hans Jonas fera un pas de plus dans les années soixante avec son ouvrage Le phénomène de la vie, en faisant de la mobilité, plus que de la seule motricité en interaction spatiale avec le monde, la source temporelle onto- et phylo-génétique de la vitalité du vivant: les organismes unicellulaires (qui selon les classifications naturalistes mentionnées plus haut incluent d'ailleurs des vivants non-«animaux ») sont d'après lui dotés d'une poussée archaïque qui alimente leur auto-développement. À cet égard, vivre (être mobile) auto-antécède connaître (percevoir), mais aussi se déplacer (être moteur/en mouvement). Pour Jonas, l'ensemble du processus de l'évolution du vivant se nourrit de cette pulsion mobile, initiale et réitérée, qui est ce qui lui confère une énergie de fond pour découvrir de nouveaux territoires, pour accueillir d'autres êtres et s'ouvrir à des événements imprévus ${ }^{26}$. Cela ne correspond pas à un instinct (de survie), mode relativement physico-mécanique de cette poussée pulsionnelle, mais davantage à ce que Jonas ${ }^{27}$ appelle un "désir ${ }^{28} \mathrm{ou}$, sur les traces du philosophe grec contemporain Christos Yannaras, un « erôs ».

\footnotetext{
${ }^{26} \mathrm{H}$. Jonas, «Les fondements biologiques de l'individualité », in Études phénoménologiques, Phénoménologie et philosophie de la nature, no 23-24, 1996, p. 102-103.

${ }^{27}$ H. Jonas, Évolution et liberté, Paris, Payot \& Rivages, 2000, p. 51 : «Si le désir aiguillonné par le besoin est la condition fondamentale de la mobilité, alors la poursuite (aller vers l'objet) représente le mouvement originel. C'est aussi là qu'apparait pour la première fois la différence entre l'animal et la plante : elle consiste en l'insertion d'un écart entre pulsion et satisfaction [...]. La perception à distance est obligatoire pour le repérage d'un tel but ».

${ }_{28}$ Cf. aussi, à propos de la distinction entre instinct, pulsion et désir, Alter no 9, La pulsion, et en particulier, N. Depraz, «Pulsion, instinct, désir. Que signifie Trieb chez Husserl ? - À l'épreuve des perspectives de Freud, Merleau-Ponty, Jonas et Scheler ».
} 
Francisco Varela enfin, à travers sa trajectoire globale de pensée, prolongera ces avancées majeures en direction de ce qu'il nomme un « radical embodiment» du vivant ${ }^{29}$ : de von Uexküll, il reprend la dynamique sensori-motrice interactive et récursive du vivant et de son monde environnant, en opère la refonte à travers son modèle de l'autopoièse avec $\mathrm{H}$. Maturana et, plus particulièrement, depuis son expression de «couplage structural» (estructural acoplamiento), qui fait du vivant auto-poiétique un être caractérisé par une dynamique circulaire de co-construction du monde et de lui-même et doté d'une autonomie foncièrement relationnelle, à savoir à ce titre résolument anti-kantienne ${ }^{30}$. Aussi émettra-t-il toujours des réserves à l'égard de l'idéal holiste de von Uexküll, et ce, en faveur de l'existence précaire du vivant. C'est pourquoi F. Varela verra le réductionnisme comme le holisme comme deux postures opposées mais coïncidant sur leur prétention unilatérale à décrire le vivant depuis un point de maîtrise,

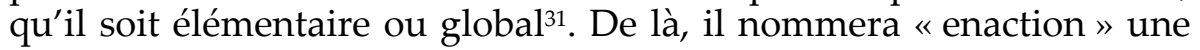
telle dynamique d'émergence qui préserve l'irréductibilité du vivant comme un soi mobile s'auto-organisant, et ce, par contraste avec une conception représentationaliste rémanente ${ }^{32}$. Sa lecture attentive de Jonas $^{33}$ le conduit à approfondir son entente enactiviste du vivant, déjà marquée en 1989 du sceau d'une «dérive naturelle » réceptive, par opposition à toute entente adaptationniste trop activiste, en situant l'expérience de l'incarnation du vivant au cœur de la précarité de la vie, c'est-à-dire de l'imminence toujours présente de la mort ${ }^{34}$. En ce sens, l'« incarnation radicale » ouvre sur l'indétermination des

\footnotetext{
${ }^{29}$ F. J. Varela et E. Thompson, «Radical Embodiment : neural dynamicqans consciousness », in Trends in Cognitive Sciences, 2001, Trends in Cognitive Sciences, 5, p. 418-25.

${ }^{30} \mathrm{H}$. Maturana et F. J. Varela, De Maquinas y seres vivos : una theoria sobre la organizacion biologica, Santiago, Editorial Universitaria, 1973; version anglaise: Autopoiesis and Cognition: The realization of the living, Boston, D. Reidel, 1980 ; traduction française, L'Arbre de la Connaissance, Paris, Addison-Wesley France, 1994 ; F. J. Varela, H. Maturana, R. Uribe, «Autopoiesis: The organization of living systems, its characterisation and a model », in Biosyst 5, 1974, p. 187-196.

${ }^{31}$ F. Varela et J. Goguen, "The arithmetic of closure ", in éd. R. Trappl, Progress in Cybernetics and Systems Research, New York, Wiley Hemisphere, vol. 3, p. 48-63.

32 F. Varela, E. Thompson, E. Rosch, The Embodied mind, MIT Press, 1989, trad. L'inscription corporelle du vivant, Paris, Seuil, 1991.

${ }^{33}$ H. Jonas, Le phénomène de la vie, op. cit., p. 7-26.

${ }^{34}$ A. Weber et F. J. Varela, «Life after Kant: Natural Purposes and the autopoietic foundations of biological individuality ", Phenomenology and the Cognitive Sciences, 1 (2), Heidelberg, Springer, 2002, p. 97-125. On soutient parfois la thèse que la position ontologique de F. Varela, de type naturaliste, est incompatible avec l'existentialisme éthique de H. Jonas. Ce serait oublier que la position éthique et existentielle de Varela dans Quel savoir pour l'éthique?, Action sagesse, cognition (1997), Paris, La Découverte, 2004 notamment, témoigne d'un investissement axiologique qui permet de rejeter une ontologique naturaliste au sens étroit.
} 
événements à venir, la passivité réceptive à l'égard de l'autre et la dynamique d'émergence auto-antécédante de la surprise ${ }^{35}$.

Quoi qu'il en soit, le mode de croissance général de l'animal (qu'il soit marqué par le déplacement moteur, l'imminence du mourir ou la réceptivité) conduit à mettre en avant l'avancée vers l'autre (marche, ouverture, exposition). La définition des êtres vivants animaux comme êtres se mouvant semble donc devoir être articulée plus précisément en y adjoignant une composante originairement relationnelle. Yannaras, philosophe grec contemporain, emploie dans son livre, Variations sur le cantique des cantiques, le beau terme d'erôs pour décrire la quête, fondé sur le désir, de la relation à l'altérité : "C'est sûrement dans l'erôs que convergent et s'accomplissent l'effet naturel et l'effet relationnel. C'est pourquoi l'erôs confirme également l'altérité, révèle le sujet. Il est la tension suprême de l'existence, le fil qui permet de sortir de l'énigme de la mortalité. Si le moi intime conscient, ou notre âme, émerge et s'affirme dans l'erôs, alors il existe seulement comme relation $»^{36}$.

\section{Végétalité37 et sécurité}

Au contraire, la vie des végétaux est, on l'a noté d'entrée de jeu, une vie placée sous le signe de l'immobilité, au sens du moins de la non-motricité. De surcroît, le trait de l'altérité relationnelle associé à la tension de l'erôs, que nous avons dégagé à propos du vivant animal, ne paraît pas caractériser le vivant végétal, dont le mode général de reproduction, non-sexué, ou encore parthénogénétique, implique un mode de vie qui est auto-affirmation de sa propre identité sans traversée constitutive et nourricière par l'autre que soi. La reproduction n'est pas confrontation exposée à l'autre, mais croissance de soi à partir de soi. On aurait affaire à un mode de fonctionnement solipsiste, qui paraît également être le mode de vie le plus confortable c'est-à-dire sécuritaire. L'absence de confrontation avec l'autre que soi

\footnotetext{
${ }^{35}$ N. Depraz, Lucidité du corps, op. cit., 2e section, "L'auto-antécédance de l'incarnation », et F. J. Varela et N. Depraz, "Au cœur du temps : l'auto-antécédance II », in Intellectica, no 36-37, 2003/1, p. 183-203.

${ }^{36}$ Ch. Yannaras, Variations sur le Cantique des Cantiques, op. cit., p. 21.

${ }^{37}$ F. Delaporte introduit le terme de "végétalité » dans son ouvrage Le Second Règne de la nature : essai sur les questions de la végétalité au XVII siècle, préface de Georges Canguilhem, Paris, Flammarion, 1979, avec le sens de premier degré, le plus simple, de la vitalité, à savoir l'ensemble des traits physiologiques communs aux végétaux et aux animaux, mais qui existent seuls chez les végétaux.
} 
va de pair avec l'absence du danger encouru dans la perte de son identité, c'est-à-dire dans l'altération de soi.

Aussi les vivants végétaux mènent-ils une vie caractérisée par le repos. Selon von Uexküll, le développement des végétaux se fait par degrés et se trouve lié à un certain endroit. L'auteur prend l'exemple de l'écorce du chêne: "De ce gland sortiront des cellules variées, dont les unes formeront des racines souterraines, les autres les branches et leurs toits de feuilles, selon une règle de développement caractéristique du chêne ${ }^{38}$. Aussi le chêne fait-il usage de l'endroit où il est de façon à croître sans avoir à se mouvoir ou à se déplacer. Dans Le Phénomène de la vie, Jonas montre à son tour de façon remarquable comment les plantes développent une telle aptitude: «La distinction entre relation immédiate et relation médiate à l'environnement coïncide avec la distinction entre plantes et animaux et doit ainsi être liée à la différence de base de leur mode de métabolisme. Par sa capacité à synthétiser des matières inorganiques directement en en faisant des composants organiques, la plante est à même de tirer sa substance de la provision minérale quotidienne disponible dans le sol, tandis que l'animal doit dépendre de la présence non-assurée de corps organiques hautement spécifiques et nonpermanents. De plus, l'absorption de nourriture solide exigée par le mode de nutrition de l'animal, par contraste avec l'absorption simplement osmotique d'éléments nutritionnels dissous par les plantes, implique l'interposition d'une stade auxiliaire de type "mécanique » (lié au transport, à la cachette) qui s'oppose à l'étape directe, chimique de l'appropriation métabolique. Sur ce plan, la plante manifeste une supériorité plutôt qu'une déficience en comparaison avec les animaux. Mais la possession de ce pouvoir unique de synthèse directe, et l'auto-suffisance qu'il permet, est la vraie raison de l'absence de ces autres traits que les animaux se sont vus contraints à développer en vertu de leur mode plus précaire de métabolisme $»^{39}$. Ou encore : «En termes de stricte sûreté biologique, les avantages de la vie animale sur la vie végétale sont hautement problématiques, et on ne saurait les faire valoir à $n^{\prime}$ importe quel prix ${ }^{40}$.

Ainsi, en premier lieu, la vie des végétaux est caractérisée par une stabilité qui est liée à leur mode assuré de nutrition, à savoir directement issu du sol inorganique. Bref, en conformité avec la distinction naturaliste entre auto- et hétéro-trophie mentionnée plus haut, l'im-

\footnotetext{
38 Von Uexküll, op. cit., p. 115.

${ }^{39}$ H. Jonas, op. cit., "Se mouvoir et ressentir : sur l'âme animale », p. 103.

${ }^{40}$ H. Jonas, op. cit., p. 106.
} 
médiateté et la présence permanente est ce qui constitue la sécurité des végétaux : la nourriture est toujours là, ils n'ont pas besoin de s'en inquiéter et, par ailleurs, son appropriation est directe, tandis que, pour les animaux, elle prend du temps. Bref, la plante est efficace là où l'animal est précaire. Ou encore: la sécurité est synonyme de plénitude et de satisfaction, tandis que l'absence, le vide ou la différence créent de l'instabilité et de l'insécurité.

\section{Chiasme et inversion sous la dualité : de la polarité risque moteur/sécurité immobile à la dynamique de l'anxiété animale et de la croissance végétale}

D'où la tendance des animaux à développer davantage de stabilité dans leur mode de vie. Étant exposés à des dangers, à l'absence d'assurance quant à la nourriture, c'est-à-dire à la précarité de leur survie, et étant ainsi sujets à l'anxiété liée à la perte, à l'absence et à la dynamique du manque inhérente au désir, ils finissent par rechercher une vie qui les sécurisera : "L'existence mobile est agitée et anxiogène : la vie des plantes n'est rien de tel $»^{41}$. Au contraire, les végétaux développant une satisfaction élevée et des automatismes efficaces qui leur "épargne la nécessité de se mouvoir » ${ }^{42}$, ils sont également à même de créer une autre sorte de liberté : «La capacité à aller dehors en quête de nourriture répond simplement à la nécessité que le type de métabolisme impose à l'animal et dont la plante est affranchie ${ }^{43}$. Une telle liberté à l'égard de la nécessité de la nature va de pair avec un autre trait que nous aborderons plus tard, la spontanéité.

Bref, la distinction initiale qui associe l'animal au risque et le végétal à la sécurité tient à un critère naturel rémanent emprunté à l'observation externe du mouvement. Or, si le vécu interne de l'animal tend depuis l'anxiété motrice à la stabilité, à l'assise, on peut présumer en chiasme que la dynamique interne du végétal ressortit, depuis l'immobilité, à une forme de mobilité d'un autre type, emprunté à la croissance générative plus qu'au déplacement bien entendu. Il y a sinon une inversion du moins un retournement des critères, dès lors que l'on passe de l'observation externe à l'appréhension interne du vécu kinesthésique ou de la dynamique générative. Une telle conversion du regard rend les critères du vivant plus perméables

${ }^{41}$ Idem.

${ }^{42}$ Idem.

${ }^{43}$ Idem. 
les uns aux autres, et conduit à prendre en considération en retour le type de mobilité du végétal et la forme de stabilité de l'animal.

\section{A. L'animal et la recherche d'une vie stable}

Si l'on regarde de plus près les mouvements continuels de l'animal, ce qui apparaît, c'est sa capacité à les synchroniser de façon à les faire servir à chaque fois à un objectif particulier. La motricité de l'animal n'est donc pas une mobilité incessante et non-orientée. Par ces mêmes mouvements, l'animal s'efforce de maîtriser ce qui lui échappe de façon irréductible. En d'autres termes, si vivre, c'est faire l'épreuve de risques inévitables, au final, ces derniers sont toujours calculables. Vous vous donnez des possibilités d'action, mais vous savez comment évaluez dans quelle mesure vous pouvez les accomplir.

Plus concrètement, les animaux vivent et se meuvent dans un environnement délimité, dont la proximité permet savoir-faire et familiarité. Il y a là des correspondances intéressantes avec la façon dont les êtres vivants sont progressivement devenus sédentaires au cours de la longue histoire de leur devenir-humain. À cet égard, la sédimentation individuelle et l'implantation historique convergent dans une pulsion similaire à créer de la stabilité par l'assise: "sedere ", s'asseoir, être assis, n'est-ce pas la racine latine commune des deux processus de "repos »: " sédimentation », "sédentarisation ». Pour pouvoir s'asseoir (y compris dans le cadre de la «méditation assise »), encore faut-il être debout et en mouvement.

Être en repos en s'asseyant, telle est l'expérience suprême de la stabilité, située notons-le à l'opposé de la mort, dans la mesure où elle permet au vivant d'expérimenter ses propres limites. Mais les limites peuvent devenir des limitations, et l'assise une immobilisation porteuse de mort. Aussi la stabilité est-elle toujours au fond ambivalente, soit expérience d'assise dans le repos, soit contre-expérience de vie rigidifiée.

\section{B. La croissance du végétal}

À l'inverse, le végétal, sans se déplacer, développe des aptitudes à engendrer depuis lui-même une forme de liberté qui prend la forme d'un mouvement interne, auto-mouvement synonyme de spontanéité, mais aussi règle interne que lit littéralement l'auto-nomie. 


\section{1) Spontanéité et satisfaction}

Lisons encore ce que nous dit von Uexküll : "Nous savons que dans le gland se trouve en puissance les organes qui permettront au chêne de soutenir sa lutte vitale contre les mille actions diverses du monde extérieur. Nous voyons en esprit le chêne futur lutter contre la pluie future, la tempête à venir et le soleil de demain. Nous le voyons résister aux étés et aux hivers ultérieurs ». Ainsi, la croissance végétale est un auto-développement qui confère à ce dernier une forme de spontanéité (du latin sponte: de soi-même, de façon autonome). Un grand nombre des actions du végétal sont mises en œuvre pour favoriser une telle autonomie : "Pour pouvoir répondre à toutes les actions du monde extérieur, les cellules du gland devront se diversifier en organes, en racines, en branches et en feuillage qui captera les rayons du soleil et dont les feuilles suivront comme de légers fanions le vent auquel résistera les branches noueuses. En même temps, le feuillage servira de parapluie qui dirigera la précieuse humidité du ciel vers les fines racines souterraines. Les feuilles renfermeront de la chlorophylle, qui utilisera les rayons solaires pour changer comme par magie leur énergie en substance». Ainsi, le végétal est apte à inventer de nouvelles actions de façon à éviter les perturbations du monde extérieur, ce qui signifie qu'il possède un grand nombre de ressources et de potentialités vitales. Von Uexküll insiste lui-même sur l'autonomie du végétal dans le sens de son automotion: "Toutes ces actions futures, que le chêne aura à subir, sont hors d'état d'influencer causalement son développement ${ }^{44}$. Le végétal crée ainsi en lui-même la possibilité de ne pas dépendre de causes extérieures, façonnant ainsi sa propre auto-causalité.

Une telle compréhension du vivant en tant qu'être doté de spontanéité autonome est noté par certains auteurs contemporains ${ }^{45}$, mais elle ne paraît pas être considérée comme un trait caractéristique des végétaux. À ma connaissance, Jonas est le seul auteur qui ait souligné de façon remarquable l'inventivité unique des végétaux, et ceci n'est pas incompatible avec l'accent qu'il porte par ailleurs sur leur "sûreté » nutritionnelle, car les deux traits contribuent à l'accomplissement de la vie végétale: "Avec les racines, les plantes ont "inventé" le moyen le plus efficace d'exploiter les avantages inhérents à l'organisme qui opère la photosynthèse [...]. À travers leur contact continu avec la source d'approvisionnement, la relation entre l'orga-

\footnotetext{
${ }^{44}$ Von Uexküll, op. cit., p. 115.

${ }^{45}$ M. Sheets-Johnston, The primacy of movement, op. cit. ; E. Thompson \& F. J. Varela, Why the mind is not in the brain, Cambridge MA, Harvard U. P., 2012.
} 
nisme et l'environnement fonctionne de façon automatique et aucun autre appareil d'adaptation à des changements à court terme n'est nécessaire [...]. Comme la satisfaction est contemporaine de l'activité vitale, il n'y a aucun intervalle dans lequel le besoin pourrait venir à être ressenti par lui-même et où l'activité devrait être opérée d'ellemême, comme la marque d'une appétition $»^{46}$.

La poussée créative des végétaux tient à leur accomplissement en plénitude. Cela a pour conséquence que leur expérience é-motionnelle n'est pas une réactivité à un désir inaccompli, comme c'est le cas des animaux.

2) Végétalité et émotionnalité

Être touché, c'est être mû. Or, l'é-motion, cela signifie, littéralement, "mouvoir ou être mû hors de soi-même » (ex-movere) ${ }^{47}$. C'est ce qui semble être caractéristique des animaux plus que des végétaux, lesquels sont incapables de sortir d'eux-mêmes. On associe à cet égard souvent l'émotion à la distance : «[...] le désir présente l'objet comme "pas-encore là mais à venir" : la mobilité guidée par la perception et poussée par le désir transforme le "là-bas" en un "ici" et le "pas encore là" en un "maintenant". Cela, sans la tension conditionnée par la médiation animale, où l'animal s'émancipe de son immersion dans une fonction organique aveugle et reprend de luimême une fonction : ces fonctions, ce sont les émotions. L'être animal est ainsi essentiellement un être passionné $\gg^{48}$.

Si l'émotion-passion caractérise l'animal, cela ne signifie pas que le végétal ne dispose pas d'une autre forme d'émotionnalité. Lisons ce que nous en dit parallèlement Hans Jonas : "L'émotion ne peut être identifiée à partir d'un organe externe ni faire son entrée sur le plan physique sous la forme d'un compte rendu ; une telle invisibilité ou complète intériorité $[\ldots]$ fait qu'on pourrait penser pouvoir s'en passer dans une description du comportement corporel [...] $»^{49}$. C'est là qu'il convient de distinguer l'émotion-passion, qui est réaction externalisée, et l'é-motion-fluctuation, qui répond à la dynamique globale du ressenti. C'est pourquoi cette émotionalité va remarquablement de pair avec la végétalité, ou encore avec ce que l'on pourrait nommer le « système végétal », lequel se déploie dans sa croissance à

\footnotetext{
${ }^{46}$ H. Jonas, op. cit., p. 102.

${ }^{47}$ Voir sur ce point, N. Depraz, «Délimitations de l'émotion : pour une phénoménologie du cœur », Alter no 8, L'émotion, Dijon, Alter, 1999.

${ }^{48}$ H. Jonas, op. cit., p. 105-106.

${ }^{49}$ H. Jonas, op. cit., p. 100.
} 
l'insu de l'observation externe et revêt ce faisant une forme d'immanence invisible.

C'est pourquoi, plutôt que maintenir une dualité externe entre deux émotions répondant de façon exclusive à deux types de vivants, on fera l'hypothèse que la dimension de la végétalité au sein même $d u$ vivant animal et humain se développe comme une dynamique radicale de vitalité interne: "The affective force manifests as a rapid, dynamical transformation from tendency to salience, involving one's entire leib (lived body) as a complex [...] the gamut of autonomic action such as respiratory, heart rate, endocrine secretion, etc. as well as the ancestral motor pattern involved in posture and movement [...] a feeling grounded in the body's responsive repertoire $»^{50}$. C'est ainsi que le nom de "valence », couramment utilisé en neuro-psychologie des émotions pour décrire la polarité émotionnelle +/- (agréable/désagréable, plaisant/déplaisant) et initialement introduit par K. Lewin pour décrire le comportement du vivant comme « champ de forces ${ }^{51}$ a été donné par Depraz et Varela à ce mouvement vital interne, également appelé «fluctuation primordiale » : "The gradually emerging change $[. .$.$] is an affect-emotion in the self-movement of the flow, of$ the temporal stream of consciousness $»^{52}$.

\section{3) L'ambivalence de la flexibilité : permissivité et inertie}

Bref, le mode végétal de la vie se caractérise pas trois traits principaux: 1) a spontanéité entendue comme satisfaction directe, 2) l'émotionnalité-fluctuation, et à présent, 3) la flexibilité jusque dans son retournement en inertie, où végétal se métaphorise en "végétatif ». Ce dernier trait ambivalent, que je vais aborder pour finir, touche à la qualité de souplesse qui caractérise le vivant végétal, orthogonale à toute loi synonyme de limite et d'interdit. Comme

\footnotetext{
${ }^{50}$ F. J. Varela \& N. Depraz, «At the source of time : valence and the constitutional dynamics of affect», in Ipseity and Alterity. Arob@se: An electronic Journal, 2000, Copie papier, Presses Universitaires de Rouen, 2001. Republié par E. Thompson dans le Journal of Consciousness Studies, Emotions and the First person perspectives, Imprint Academic, 2008.

${ }^{51}$ Le concept de valence a été introduit par Kurt Lewin dans les années 1930 afin de designer le champ de forces qui explique la dynamique de comportement, soit les mouvements d'attraction ou de répulsion de l'individu. Ce sont ainsi les valences qui configurent et définissent les régions dans l'espace de vie. Pour Lewin, le champ de forces se caractérise par une tendance à l'équilibre et c'est cet aspect qui distingue la position de cet auteur de celle de Varela et Depraz, lesquels mettent l'accent sur sa dimension de fluctuation. (Principles of topological psychology, New York/London, McGraw-Hill, 1936.)

52 F. J. Varela \& N. Depraz, art. cit. ; voir sur ce point D. Rudrauf et al., «From autopoiesis to neurophenomenology : Francisco Varela's exploration of the biophysics of being ", in Biological Research 36, 2003, p. 27-65, ici p. 59.
} 
Pascal très tôt le souligne dans une pensée célèbre, le roseau toujours plie mais jamais ne rompt. La vie végétale se caractérise par une propension remarquable à l'adaptabilité, c'est-à-dire de flexibilité53.

La flexibilité du végétal a cependant des revers, qui répondent à des tendances internes à sa dynamique : 1) l'adaptabilité peut se faire permissivité, ce qui crée, en lien avec le critère $n^{\circ} 1$ de la satisfaction immédiate spontanée, un mode de relation osmotique et quasifusionnel. D'où la contre-tendance utile du manque lié au désir, et de la loi, qui pose le cadre ; 2) le fonctionnement invisible et du coup endogène du végétal peut engendrer un mode d'être qui tend à l'automatisme et transporte le végétal vers le "végétatif ». Plus qu'une métaphore qui aurait un corrélat anthropologique fixé dans le vieillissement de l'organisme ou dans sa simple inertie corporelle ${ }^{54}$, la part «végétative » du vivant relève cependant d'une dimension de « recyclage » nécessaire à sa capacité d'invention.

\section{Conclusion : la réceptivité du vivant}

Les différentes étapes de l'analyse de la vie végétale au prisme de la polarité en chiasme du risque et de la sécurité (au delà de la simple distinction de la motricité et de l'immobilité) conduisent pour finir à proposer une entente du vivant placée sous le signe de la réceptivité.

On peut ainsi mesurer pour conclure différentes conceptions philosophiques du vivant à cette aune : 1) la discontinuité des modes d'être posée par Heidegger dans les Grundprobleme der Metaphysik (1929) est fondée sur un principe de séparation entre les modes de relation au monde : le minéral, sans monde (weltlos), l'animal, pauvre en monde (weltarm), l'humain, formateur de mode (weltbildend). Une telle délimitation conduit à accorder axiologiquement un mode de réceptivité plénier à l'humain, comme si animaux et végétaux (d'ailleurs non-distingués en propre, ce qui est fort instructif) en étaient les parents pauvres. À l'encontre de cette vision étroite du vivant, quelques conceptions du vivant entrent en résonance avec la vie entendue comme réceptivité : 1) la solidarité entre règnes (animal et végétal), dont les descriptions naturalistes attestent via l'identification de mixtes inclassables, a été bien montré par von Uexküll en tant

\footnotetext{
${ }^{53}$ N. Depraz, "Erôs et relation. Puissance générative et engendrement mutuel des pensées de Michel Henry et Christos Yannaras ", Alter no 18, Erôs, Dijon, Alter, 2011, et N. Depraz et F. Mauriac, "Phénoménologie féminine de l'erôs", Colloque "Femme, Erôs, philosophie ", Université de Louvain-la-neuve, 6 décembre 2011, Actes à paraître.

54 J.-P. Sartre, L'être et le néant, Paris, Gallimard, 1945, IV, I, 1.
} 
qu'expression directe du sens réceptif de la circularité : il y a là une méthodologie holiste dans la veine ultérieure de Goldstein dans l'Aufbau des Organismus et de von Weizsäcker dans Der Gestaltkreis. Cependant, l'accent porté sur l'exposition à l'altérité introduit une sérieuse limitation à la pertinence complète du holisme, et permet de rendre compte de la fragilité de l'être vivant ; 2) la complémentarité entre animaux et végétaux chez $\mathrm{H}$. Jonas montre comment chacun possède à la fois force et faiblesse: tandis que le végétal est doté d'une efficacité due à son sens de la sécurité, l'animal se caractérise par un sens passionné du mouvement; mais une telle passion est également source d'instabilité, alors que l'environnement sécurisant du végétal contient à son tour en germe une tendance mortifère à l'inertie.

Être un vivant, n'est-ce pas être un mixte d'exposition et de stabilité, selon une signature qui singularise chacun selon son coefficient de tendance végétale ou animale? 